\title{
Preparação psicológica nos serviços de psicologia pediátrica dos hospitais universitários públicos federais ${ }^{1}$
}

\author{
Psychological preparation in the area \\ of pediatric psychology at federal \\ public university hospitals
}

\author{
Fernanda Nascimento Pereira DOCA ${ }^{2}$ \\ Áderson Luiz COSTA JUNIOR ${ }^{3}$
}

\section{Resumo}

A preparação psicológica tem sido recomendada pela literatura em função da probabilidade de produzir aumento dos índices de adesão ao tratamento e redução de ansiedade. Todavia, sua utilização requer intervenções mais sistemáticas que conduzam a resultados mais consistentes. Este estudo caracteriza as intervenções de preparação psicológica desenvolvidas em serviços de psicologia pediátrica de hospitais universitários públicos federais brasileiros. Utilizou-se um questionário eletrônico autoaplicável, preenchido via Internet. Participaram da pesquisa 25 psicólogos vinculados a 11 hospitais. Embora todos os participantes refiram-se à utilização de preparação psicológica, 10 adotam outras denominações, e apenas nove efetuaram algum tipo de avaliação de resultados. Verificou-se, entre os participantes, ausência de consenso teórico ou metodológico no tocante à nomenclatura, aos objetivos, às técnicas e aos procedimentos de execução. Sugere-se o desenvolvimento de pesquisas sobre preparação psicológica, utilizando-se técnicas e/ou recursos variados que apontem relações funcionais mais precisas entre intervenções e efeitos, a fim de estabelecer protocolos de ações práticas.

Unitermos: Hospitais universitários. Preparação psicológica. Psicologia pediátrica.

\begin{abstract}
Literature has recommended psychological preparation due to the likelihood of increasing treatment adhesion rates and reduced anticipatory anxiety. However, its use requires interventions that are more systematic which lead to more consistent results. This study characterizes psychological preparation interventions developed in pediatric psychology services in Brazilian federal public university hospitals. We used a self-administered electronic questionnaire completed via the Internet. Twenty-five psychologists linked to 11 hospitals participated in the study. Although all participants have referred to the use of psychological preparation, 10 adopted different designations, and only nine conducted any kind of evaluation results. We have not determined a theoretical or methodological consensus regarding nomenclature, objectives, techniques and execution procedures amongst the participants. This study claims that there is a need to develop scientific research into psychological preparation which uses different techniques and/or resources and which aims to demonstrate more precise functional relationships between intervention and effects, in order to establish guidelines for practical action.
\end{abstract}

Uniterms: Hospitals university. Psychological preparation. Pediatric psychology.

\section{४}

1 Artigo elaborado a partir da dissertação de F.N.P. DOCA, intitulada "A psicologia pediátrica em hospitais universitários brasileiros". Universidade de Brasília, 2009.

2 Doutoranda, Universidade de Brasília, Programa de Pós-Graduação em Processos de Desenvolvimento Humano e Saúde. Brasília, DF, Brasil.

3 Universidade de Brasília, Instituto de Psicologia. Campus Universitário Darcy Ribeiro, Prédio Minhocão, 70910-900, Brasília, DF, Brasil. Correspondência para/Correspondence to: A.L. COSTA JUNIOR. E-mail: <aderson@unb.br>. 
No intuito de reduzir a ocorrência, a magnitude e o impacto das repercussões adversas da hospitalização e/ou do tratamento médico infantil (Crepaldi, Rabuske \& Gabarra, 2006; Doca \& Costa Junior, 2007), a psicologia pediátrica tem desenvolvido uma série de procedimentos de intervenção, utilizando-se de variadas técnicas e recursos, dentre os quais se pode destacar a preparação psicológica.

Embora a preparação psicológica seja objeto de pesquisa desde as décadas de 1960 e 1970 (Salmon, 2006), e se observe consenso sobre a necessidade de que toda criança receba algum tipo de preparação para a exposição às experiências vivenciadas durante a hospitalização e o tratamento médico (Roberts, 2003), até o presente momento não há, nem na literatura científica, nem na prática profissional, uma definição clara e consensual dessa intervenção. A definição de Quiles e Carrillo (2000), adotada pelo presente estudo, por exemplo, é a de um conjunto de procedimentos técnicos cujos objetivos gerais são prevenir e/ou aliviar as reações emocionais advindas da situação de internação hospitalar e facilitar a adaptação comportamental ao contexto.

De forma específica, a preparação psicológica pode visar, entre outros aspectos: (a) reduzir o medo, a ansiedade antecipatória e/ou o distress (Broering \& Crepaldi, 2008; LeRoy et al., 2003; Patel et al., 2006); (b) desenvolver estratégias de enfrentamento mais eficientes ao tratamento e aos episódios que o compõem (Franck \& Jones, 2003); (c) aumentar os índices de adesão ao tratamento (Caminiti, Scoditti, Diodati \& Passalacqua, 2005); (d) promover a cooperação e o ajustamento comportamental durante e após a execução de procedimentos médicos (LeRoy et al., 2003; Soares \& Bomtempo, 2004); e (e) auxiliar na redução/manejo da dor experienciada pela criança/adolescente (Blount, Piira, Cohen \&Cheng, 2006; Uman, Chambers, McGrath \& Kisely, 2008).

Intervenções dessa natureza pressupõem a antecipação de um fato/evento, devendo, portanto, ser realizadas em momento anterior à sua ocorrência. Assim, podem anteceder o período de hospitalização, o momento da admissão hospitalar e a realização de exames e procedimentos invasivos. Todavia, Doca e Costa Junior (2007), em revisão da literatura especializada, apontam que a preparação psicológica é mais frequentemente executada antes de exames e procedimentos invasivos, provavelmente por se considerar o alto potencial aver- sivo desses eventos (Blount et al., 2006) e a alta frequência de comportamentos indicadores de sofrimento (Costa Junior, 2001), ansiedade e medo deles decorrentes, tanto para as crianças quanto para seus familiares/acompanhantes, o que intensifica a dor sentida pelo paciente e interfere na realização do procedimento (Uman et al., 2008).

Costa Junior (2005) observa que a preparação psicológica tende a enfatizar, de modo não exclusivo, os seguintes aspectos considerados essenciais: (a) a adaptação física e social do ambiente de cuidados (a enfermaria pediátrica, por exemplo) às necessidades psicológicas de pacientes e acompanhantes; (b) o fornecimento de informações educativas e/ou distrativas que promovam uma maior colaboração do paciente com o procedimento a ser executado; e (c) o treinamento de profissionais de saúde para a identificação de indicadores de desenvolvimento infantil/adolescente e de comportamentos de comunicação do paciente com a equipe de saúde.

Uma vez estabelecido o fato/evento para o qual o paciente necessita ser preparado psicologicamente, deve-se definir quando essa intervenção será realizada. O tempo de antecedência da preparação psicológica é uma questão polêmica na literatura, tendo em vista que, quando é realizada temporalmente muito próxima ao procedimento, pode estabelecer uma condição de ansiedade antecipatória e de distress; quando realizada muito tempo antes do procedimento, pode dificultar e até impedir a associação da criança entre os eventos (Salmon, 2006). Dessa forma, Costa Junior (2005) recomenda que o tempo de antecedência seja definido pela equipe de saúde a partir de uma avaliação das necessidades psicossociais do paciente, e que sejam considerados o custo-benefício dos procedimentos e a rotina da unidade hospitalar. Essa avaliação deve considerar, entre outros aspectos: o nível de desenvolvimento cognitivo da criança; seu temperamento; as experiências médicas anteriores (especialmente as adversas) a que a criança foi exposta; os mecanismos de enfrentamento adotados pela criança e pela família; as práticas culturais vigentes, as crenças e religião da família; a compreensão, o nível de informação e os medos relativos ao procedimento/tratamento; a existência/influência de outros estressores no tratamento (problemas financeiros, dificuldades sociais); a disponibilidade de rede de suporte social (LeRoy et al., 2003). 
Para atingir os objetivos da preparação psicológica, podem-se utilizar diferentes técnicas/procedimentos, a depender dos recursos existentes na unidade e das condições psicossociais do paciente e de seu familiar/acompanhante, averiguados na avaliação. De acordo com Quiles e Carrillo (2000), as técnicas mais utilizadas nas intervenções de preparação psicológica envolvem: (a) fornecimento de informação; (b) apresentação de recursos audiovisuais; (c) uso de brinquedos/jogos e; (d) desenvolvimento de habilidades de enfrentamento.

O fornecimento de informação oral, visual e/ou escrita é o procedimento mais utilizado desde a década de 1970 (Costa Junior, 2005), e a linguagem deve ser simples, condizente com a realidade que será vivenciada e adequada ao nível de compreensão da criança e/ou de seus familiares/acompanhantes, a fim de não gerar interpretações equivocadas (LeRoy et al., 2003).

A apresentação de recursos audiovisuais, desde material em transparências de retroprojeção até filmes editados de curta duração, além de fornecer informação acerca do procedimento/tratamento, pode incluir orientações sobre técnicas de enfrentamento, tais como relaxamento e visualização. Embora imagens sejam potencialmente atrativas e didáticas, os efeitos desse recurso podem ser diferenciados em crianças com experiência adversa de hospitalização/tratamento infantil, bem como em crianças pequenas, podendo sensibilizá-las e Ihes aumentar o nível de ansiedade antecipatória (LeRoy et al., 2003).

Os jogos e as brincadeiras são técnicas utilizadas para estimular a expressão afetiva e cognitiva da criança/adolescente com relação a eventos estressores. Brinquedos relacionados ao contexto hospitalar (seringa, estetoscópio, entre outros) são frequentemente utilizados por proporcionarem a familiarização da criança com elementos da situação potencialmente estressante a ser enfrentada (Yamada \& Bevilacqua, 2005). De forma semelhante, jogos eletrônicos e videogames têm sido muito utilizados com o objetivo de facilitar a aprendizagem de habilidades de enfrentamento de situações que envolvam stress e ansiedade (Franck \& Jones, 2003; Patel et al., 2006) e que simulem situações reais com grande veracidade.

Por fim, o desenvolvimento e/ou treino de habilidades de enfrentamento, efetuado por meio de técnicas como o relaxamento, a imaginação ativa, o controle da respiração, entre outras, auxiliam o controle das emoções, especialmente da ansiedade (Ribeiro, Tavano \& Neme, 2002). Essas técnicas tendem a aumentar a eficácia dos programas de preparação psicológica, particularmente quando a criança/adolescente já tem experiência com tratamentos médicos (LeRoy et al., 2003).

A respeito das técnicas utilizadas durante as intervenções de preparação psicológica, Caminiti et al. (2005) ressaltam que a probabilidade de se alcançarem resultados mais efetivos é maior quando as técnicas são associadas entre si, são executadas por equipes multiprofissionais e requerem a participação ativa das crianças e seus familiares/acompanhantes (LeRoy et al., 2003).

Apesar de a literatura apontar resultados potencialmente positivos com a prática da preparação psicológica, a falta de uma abordagem sistemática e organizada limita as conclusões sobre sua eficácia (Uman et al., 2008), especialmente porque pouco ainda se sabe sobre os mecanismos que podem alterar os processos psicológicos básicos de crianças/adolescentes em tratamento médico e expostas a procedimentos de preparação psicológica (Salmon, 2006). Essa situação limita o alcance das pesquisas em termos da aplicabilidade da intervenção em larga escala, dificulta a tomada de decisão do pesquisador acerca de seu uso, bem como a implementação sistemática de programas dessa natureza, de modo que a prática profissional ainda não acompanha, de forma consistente, alguns dos resultados apontados pela literatura na área (LeRoy et al., 2003; Salmon, 2006).

Considerando a preparação psicológica, especialmente no que se refere: (a) aos resultados positivos alcançados com a utilização dessa intervenção, em contraposição às lacunas existentes na área; (b) à descrição metodológica insuficiente de programas dessa natureza; e (c) à limitação das pesquisas acerca da eficácia de seus resultados (Broering \& Crepaldi, 2008), realizou-se este estudo com o objetivo de caracterizar as intervenções de preparação psicológica desenvolvidas por psicólogos atuantes em unidades pediátricas de hospitais universitários públicos federais.

\section{Método}

Este estudo é parte de uma investigação mais ampla que analisa a condição de desenvolvimento da 
psicologia pediátrica no Brasil, a partir da atuação profissional dos psicólogos em serviços de psicologia pediátrica nos hospitais universitários públicos federais. Este artigo se refere, especificamente, às intervenções de preparação psicológica realizadas pelos psicólogos. É caracterizado como um estudo exploratório, descritivo e de caráter qualitativo.

A coleta de dados foi realizada por meio de um questionário eletrônico autoaplicável, desenvolvido especificamente para este estudo, registrado e hospedado no domínio público <http://www.psicologia pediatrica.com.br>. O acesso ao instrumento era restrito, podendo ser efetuado somente por meio de usuário e de senha individuais, cadastrados e enviados por e-mail aos participantes. O instrumento era composto por 29 questões, divididas em três partes, sendo a terceira parte destinada à investigação das ações de preparação psicológica (15 questões). Devido à inexistência de uma conceituação objetiva e consensual sobre o que é preparação psicológica, utilizou-se, no instrumento, o conceito proposto por Quiles e Carrilo (2000), referido anteriormente.

Os hospitais da amostra foram selecionados a partir de amostragem não probabilística do tipo conveniência, baseada na acessibilidade das instituições e dos profissionais. Os critérios de inclusão adotados foram: (a) hospitais da rede pública vinculados às Instituições Federais de Ensino Superior (IFES), com administração direta do Ministério da Educação (MEC); (b) hospitais com atendimento pediátrico; e (c) hospitais com profissionais de psicologia atuando na área pediátrica. 0 Hospital Universitário de Brasília foi excluído da amostra, visto que os pesquisadores eram a ele vinculados.

Dessa forma, foram contatados, por meio eletrônico e/ou telefônico, 44 hospitais universitários públicos federais, a fim de se obterem informações acerca de psicólogos pediátricos atuantes nessas instituições. Entretanto, apenas 11 hospitais, distribuídos entre as cinco regiões brasileiras, responderam ao contato, disponibilizando informações de 42 psicólogos pediátricos a eles vinculados. Todos os psicólogos identificados foram contatados e convidados a participar da pesquisa, porém a participação efetiva, por meio da resposta integral ao questionário eletrônico, foi efetuada por apenas 25 82 psicólogos (taxa de retorno do questionário de 60\%).
Os participantes eram predominantemente do sexo feminino (24), com idade entre 25 e 53 anos (média de idade =38,8 anos; desvio-padrão =8,9 anos; coeficiente de variação $=23 \%$ ); a maioria tinha formação em nível de pós-graduação (10 com especialização, nove com mestrado e dois com doutorado) e atuava na área pediátrica há, pelo menos, quatro anos (17 psicólogos).

Os dados obtidos, tabulados automaticamente pelo sistema, foram analisados por técnicas de: (a) estatística descritiva, com distribuição de frequência; (b) categorização funcional de respostas (organizadas conforme os temas abordados pelos respondentes); e (c) análise de agrupamentos (análise de cluster).

O projeto de pesquisa foi aprovado pelo Comitê de Ética em Pesquisa da Faculdade de Ciências da Saúde da Universidade de Brasília, registrado sob o Certificado de Apresentação para Apresentação Ética (CAAE) n 0043.0.012.000-07.

\section{Resultados}

A caracterização das intervenções de preparação psicológica infantil foi efetuada conforme os seguintes aspectos: a utilização, ou não, de intervenções dessa natureza, seus objetivos, o momento em que eram executadas, as técnicas utilizadas, o público-alvo e os mecanismos adotados para avaliação dos resultados. (Tabela 1). Todos os 25 participantes afirmaram utilizar intervenções com propósitos semelhantes aos da preparação psicológica, tal como conceituada na pesquisa. Entretanto, 10 participantes declararam usar outras denominações, das quais: atendimento psicológico (referida por dois participantes), intervenção/apoio/acompanhamento/acolhimento psicológico, ação de natureza psicoterápica, abordagem clínica e metodologia de intervenção, referidas uma vez cada uma. Um dos participantes ainda declarou que utilizava intervenções dessa natureza, porém não as denominava por qualquer nome específico.

Apenas oito psicólogos afirmaram que desenvolviam a preparação psicológica de forma sistematizada, ou seja, a partir de procedimentos planejados, organizados e padronizados, enquanto 17 relataram que não o faziam dessa forma. Ressalta-se que o estudo não investigou a forma como essas intervenções eram sistematizadas. 
Tabela 1. Caracterização geral das intervenções de preparação psicológica. Brasília (DF), 2008 - 2009.

\begin{tabular}{|c|c|c|}
\hline Caracterização & Sim & Não \\
\hline Utilização & 25 & 0 \\
\hline \multicolumn{3}{|l|}{ A preparação psicológica é utilizada pelos psicólogos pediátricos nos hospitais universitários? } \\
\hline Denominação & 15 & 10 \\
\hline \multicolumn{3}{|l|}{ A denominação utilizada pelos psicólogos pediátricos dos hospitais universitários é "preparação psicológica"? } \\
\hline Sistematização & 8 & 17 \\
\hline \multicolumn{3}{|l|}{$\begin{array}{l}\text { A preparação psicológica é desenvolvida de forma sistematizada pelos psicólogos pediátricos dos hospitais } \\
\text { universitários? }\end{array}$} \\
\hline Especificação eventos & 20 & 5 \\
\hline \multicolumn{3}{|l|}{$\begin{array}{l}\text { Os psicólogos pediátricos dos hospitais universitários especificam os eventos do tratamento para os quais a } \\
\text { preparação psicológica é utilizada? }\end{array}$} \\
\hline Avaliação dos resultados & 9 & 16 \\
\hline $\begin{array}{l}\text { Os psicólogos pediátricos dos hospitais universitários realizam algum tipo de avaliação dos resultados das } \\
\text { intervenções de preparação psicológica? }\end{array}$ & & \\
\hline
\end{tabular}

Dentre os participantes, vinte especificaram os fatos/eventos do tratamento nos quais adotavam intervenções de preparação psicológica, sendo eles: (a) investigação e/ou revelação diagnóstica: três referências; (b) cirurgias: doze referências; (c) transplantes hepático ou de medula óssea: três referências; (d) internações prolongadas e/ou recorrentes: quatro; (e) eventos, procedimentos e/ou necessidades do tratamento (adaptação à dieta hospitalar, adesão ao tratamento, mudança de estilo de vida, procedimentos invasivos e transferência para equipe de adultos): sete referências; (f) pacientes atendidos por determinadas especialidades médicas (oncologia pediátrica, gastropediatria, otorrinolaringologia) ou acompanhados em determinadas unidade de atendimento (Unidade de Terapia Intensiva - UTI -, internação pediátrica): treze referências; e (g) terminalidade/morte: três referências.

No que tange à origem da demanda pela utilização de intervenções de preparação psicológica, 16 participantes referiram solicitação da equipe de saúde; 14 participantes referiram demanda proveniente de pacientes; 12 de familiares; 11 participantes referiram demandas próprias, e 2 participantes explicitaram outras fontes: a comunidade (rede SUS) e a direção do hospital. Vale ressaltar que 16 participantes referiram mais de uma fonte de demanda.

Quanto aos objetivos que norteavam a preparação psicológica, os participantes afirmaram utilizar a intervenção com o objetivo de informar (em 21 casos), reduzir o nível do stress (21), reduzir o medo do paciente (20), aumentar o nível de adesão ao tratamento (22), reduzir a ansiedade (22), facilitar o enfrentamento (22) e outros (4). Faz-se notar que 19 participantes afirmaram que utilizam a preparação psicológica visando a alcançar todos os objetivos anteriormente citados.

Nos serviços de psicologia pediátrica investigados, os profissionais utilizavam-se da preparação psicológica para anteceder: (a) o período de hospitalização (em 7 casos); (b) o momento da admissão hospitalar (em 17); (c) exames e/ou procedimentos médicos invasivos (em 18); (d) cirurgias (em 19); (e) o momento de alta hospitalar (em 10); e (f) outros momentos (em 6, dos quais 2 referiam-se ao período de internação de forma geral, e outros 4 ao retorno e/ou acompanhamento ambulatorial.

Os exames e/ou procedimentos médicos invasivos precedidos de preparação psicológica eram: (a) cirurgias (referidas por 8 participantes); (b) exames invasivos (por 9 participantes); (c) procedimentos invasivos (11); e (d) outros procedimentos, tais como procedimentos emergenciais clínicos, admissão em UTI por intercorrência, entre outros (referidos por nove participantes).

As cirurgias precedidas de preparação psicológica eram: ortopédicas (referidas por 7 profissionais), cardíacas (3), otorrinolaringológicas (3), neurológicas (2), transplantes (5), entre outras (hérnia, hipospádia, correção de má formação congênita etc.) - referidas, cada 
uma, por apenas um profissional. Faz-se notar que oito profissionais mencionaram realizar preparo psicológico para todos os tipos de cirurgia.

No que se refere à execução da preparação psicológica, todos os participantes (25) afirmaram que a intervenção era realizada por psicólogos, dos quais sete realizavam-na com exclusividade (sem o auxílio de outros profissionais), nove realizavam-na com outros profissionais, porém separadamente, e nove realizavam a preparação psicológica de forma integrada com outros profissionais da equipe (em caráter multi ou interdisciplinar).

Os profissionais que auxiliavam a execução da preparação psicológica de forma separada ou integrada eram: assistente social e enfermeiro (referidos, cada um, por 15 participantes), médico (relatado por 14 participantes), auxiliar de enfermagem (9), nutricionista (6), pedagogo (4) e outros (6). Esses outros profissionais foram terapeuta ocupacional e fisioterapeuta - citados separadamente por dois participantes cada um e por um profissional de forma combinada - e anestesista (relatado por um participante), que, embora seja uma especialidade médica, foi referida separadamente pelos participantes.

De acordo com 10 participantes, as intervenções de preparação psicológica eram executadas em grupo. Os outros 15 participantes não responderam a essa questão.

No que se refere às técnicas utilizadas na execução da preparação psicológica, os psicólogos foram indagados sobre nove técnicas psicológicas, comumente descritas na literatura como: (a) fornecimento de informação oral; (b) fornecimento de informação escrita (manuais, folhetos, outros); (c) fornecimento de informação por meio de recursos audiovisuais (filmes, slides, transparências, outros); (d) ludoterapia (utilização de jogos, brinquedos e atividades recreativas); (e) distração; (f) relaxamento; (g) visualização/imaginação ativa; (h) dramatização/encenação/ensaio comportamental; e (i) treino de habilidades sociais. O objetivo era verificar a frequência de utilização das técnicas; para tanto, foi empregada uma escala progressiva de quatro pontos (frequentemente, ocasionalmente, raramente e nunca), em que um equivalia a "nunca" e quatro a "frequente84 mente".
As técnicas referidas como mais aplicadas foram a "informação oral" e a "ludoterapia". A informação oral foi referida por 23 respondentes como de uso frequente e por dois como de uso ocasional. A ludoterapia, por sua vez, era utilizada frequentemente por 20 respondentes e ocasionalmente por cinco participantes.

As técnicas menos referidas foram o "treino de habilidades sociais" e a "dramatização" (13 participantes nunca utilizaram), seguidos por"visualização"(12) e"relaxamento" (nunca utilizados por 10 participantes). As demais técnicas se apresentaram distribuídas entre os quatro níveis investigados.

Após a consideração das nove técnicas (consideradas como variáveis de análise) aplicadas pelos psicólogos na execução de intervenções de preparação psicológica, foi efetuada uma análise de agrupamento para identificar, por similaridade, grupos de psicólogos que tinham características similares quanto à utilização dessas técnicas. Para viabilizar a formação dos clusters, foram excluídas as variáveis "fornecimento de informação oral" e "ludoterapia", por terem aplicação similar em todos os casos, de forma que, para gerar os clusters, foram utilizadas sete variáveis. Após análise realizada pelo software Statistical Package for Social Sciences (SPSS, versão 13.0), três clusters se destacaram no conjunto de casos (Figura 1) apresenta os clusters.

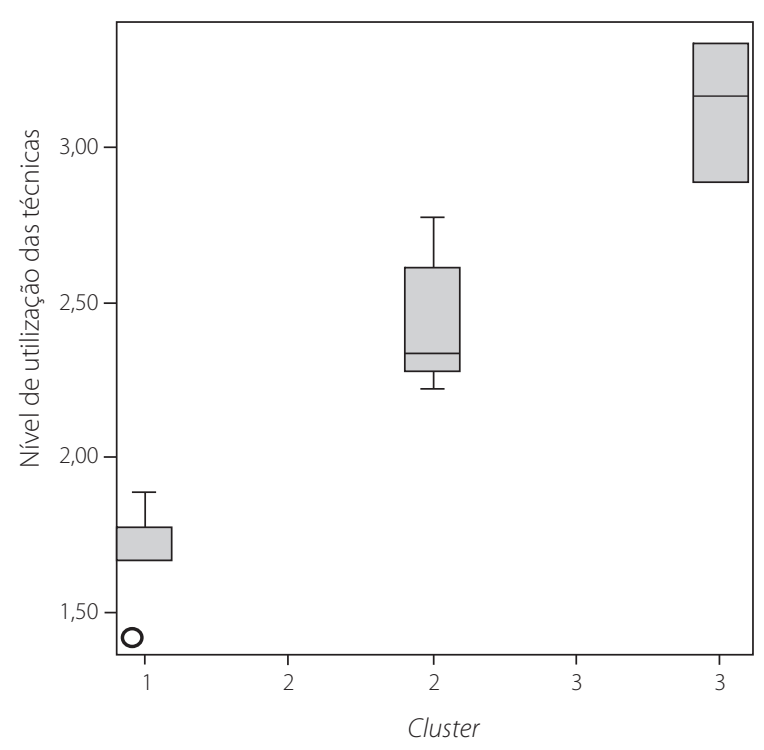

Figura 1. Análise por clusters da utilização de técnicas na preparação psicológica. 
O cluster 1 agrupou os sete casos em que os psicólogos nunca ou raramente utilizavam as técnicas listadas no instrumento de pesquisa, exceto as duas que apresentaram utilização frequente entre os respondentes (fornecimento de informação oral e ludoterapia). 0 cluster 2 foi formado por oito casos de psicólogos que apresentavam maior variabilidade no uso das técnicas, com maior índice de respostas identificadas como raramente e ocasionalmente, entre as variáveis analisadas pelas técnicas de agrupamento. O cluster 3 agrupou 10 psicólogos que utilizavam com maior frequência todas as técnicas referidas no instrumento (Tabela 2).

No que se refere à avaliação das intervenções de preparação psicológica, nove participantes afirmaram efetuar algum tipo de avaliação dos resultados. Destes, oito referiram que utilizavam: (a) ferramentas organizacionais (estatística de atendimento, relatório de atividades e avaliação institucional) - referidas por três participantes; (b) discussões de casos com a equipe - cinco participantes; (c) pesquisas (metodologia não especificada) - três participantes; (d) observação clínica não sistematizada - um participante; e (e) instrumentos sistematizados e/ou padronizados (escala de faces, roteiro de entrevista) - um participante. Foi referida, ainda, a participação em congressos e reuniões científicas. No entanto, por constituir uma consequência da avaliação, essa resposta não foi contabilizada como uma forma de proceder à avaliação.

\section{Discussão}

A partir dos resultados, observa-se que todos os participantes mencionaram o uso de intervenções que se enquadravam no conceito apresentado de preparação psicológica, corroborando o exposto na literatura acerca da importância dessa intervenção (Roberts, 2003). Contudo, uma parcela significativa dos profissionais (40\%) afirmou utilizar diferentes denominações para designar essa intervenção, e as nomenclaturas apresentadas pelos participantes eram amplas, gerais e inespecíficas. A amplitude dos termos utilizados evidencia a falta e, em contrapartida, a necessidade de definição operacional das intervenções psicológicas (mesmo as mais comumente referidas como utilizadas), bem como a uniformização da nomenclatura adotada, a fim de facilitar a comunicação e o desenvolvimento de ações conjuntas entre os próprios psicólogos atuantes na área, e destes com os outros profissionais da equipe de saúde, colaborando, assim, para o estabelecimento e para o desenvolvimento da psicologia pediátrica no Brasil.

Apesar de a preparação psicológica incluir um conjunto de intervenções muito aplicadas e pesquisadas, segundo Broering e Crepaldi (2008) ainda existem muitas lacunas, especialmente no que se refere à descrição precisa de programas dessa natureza, fato observado na pesquisa, considerando o baixo número de profissionais que desenvolvem essa intervenção de forma sistemática. Diante dessa situação, faz-se necessário o investimento no planejamento, na organização e na padronização dessa intervenção, tendo em vista que a sistematização facilita a interação com outras áreas da saúde e, consequentemente, beneficia os usuários do sistema de saúde ao melhorar a qualidade do atendimento dispensado (Gorayeb \& Guerrelhas, 2003).

Tabela 2. Análise descritiva dos clusters. Brasília (DF), 2008 - 2009.

\begin{tabular}{|c|c|c|c|c|c|c|c|c|c|}
\hline \multirow{2}{*}{ Variáveis } & \multicolumn{3}{|c|}{ Cluster 1} & \multicolumn{3}{|c|}{ Cluster 2} & \multicolumn{3}{|c|}{ Cluster 3} \\
\hline & M & $\mathrm{DP}$ & Coef. variação (\%) & M & $\mathrm{DP}$ & Coef. variação (\%) & $M$ & $\mathrm{DP}$ & Coef. variação (\%) \\
\hline Informação oral & 3,71 & 0,49 & 7,60 & 4,00 & 0,00 & 0,00 & 4,00 & 0,00 & 0,00 \\
\hline Informação escrita & 1,29 & 0,49 & 2,64 & 3,13 & 0,84 & 3,75 & 3,10 & 0,74 & 4,20 \\
\hline Informação audiovisual & 1,29 & 0,76 & 1,71 & 1,50 & 0,54 & 2,80 & 2,70 & 0,82 & 3,28 \\
\hline Ludoterapia & 3,86 & 0,38 & 10,21 & 3,75 & 0,46 & 8,10 & 3,80 & 0,42 & 9,00 \\
\hline Distração & 1,14 & 0,38 & 3,02 & 2,38 & 1,30 & 1,83 & 3,30 & 0,68 & 4,89 \\
\hline Relaxamento & 1,00 & 0,00 & 0,00 & 2,13 & 0,84 & 2,55 & 2,60 & 0,84 & 3,08 \\
\hline Visualização & 1,00 & 0,00 & 0,00 & 1,75 & 1,04 & 1,69 & 2,90 & 0,74 & 3,93 \\
\hline Dramatização & 1,00 & 0,00 & 0,00 & 1,25 & 0,46 & 2,70 & 3,10 & 0,74 & 4,20 \\
\hline Treino habilidades sociais & 1,00 & 0,00 & 0,00 & 2,00 & 1,41 & 1,41 & 2,70 & 0,82 & 3,28 \\
\hline
\end{tabular}

Coef.: coeficiente; M: média; DP: desvio-padrão. 
Os resultados apontam também que os participantes utilizam a preparação psicológica em diversos tipos de tratamentos médicos e para diferentes fatos/ eventos desses tratamentos. Todavia, pode-se notar que intervenções dessa natureza são mais comumente utilizadas antes de procedimentos invasivos, dentre eles, as cirurgias. Esse resultado é corroborado por diversas pesquisas que apontam o potencial aversivo desses procedimentos e a alta frequência de comportamentos indicadores de sofrimento, ansiedade e medo deles decorrentes, como os fatores funcionalmente responsáveis pela concentração de intervenções nesses episódios de tratamento (Blount et al., 2006; Costa Junior, 2001; Uman et al., 2008).

No que tange aos objetivos almejados com o emprego da preparação psicológica, 19 participantes afirmaram utilizar essa intervenção com todos os objetivos listados no instrumento, que se referiam aos objetivos mais comumente descritos na literatura. Contudo, os programas de preparação psicológica descritos na literatura, de modo geral, apresentam-se mais bem delimitados, tal como o proposto no estudo de Soares e Bomtempo (2004), que pretendia, com a preparação psicológica, diminuir a frequência de ocorrência de comportamentos concorrentes e aumentar os comportamentos de adesão em crianças expostas a procedimento médico de inalação. Dessa forma, mais uma vez ressalta-se a importância de definir critérios a partir dos quais psicólogos pediátricos possam pautar-se no desenvolvimento de sua rotina prática de cuidados em saúde.

Segundo os participantes da pesquisa, a preparação psicológica é executada principalmente em grupos, tendo como foco tanto o paciente quanto seu familiar/acompanhante. Sua execução é realizada sempre por profissionais de psicologia, que, muitas vezes, são auxiliados, de forma separada ou integrada, por outros profissionais, principalmente assistentes sociais, enfermeiros e médicos, o que exige habilidades e competências diferenciadas do psicólogo para o trabalho em equipe, tais como a comunicação e a justificação clara e objetiva dos procedimentos técnicos (Tonetto \& Gomes, 2007).

Com relação às técnicas utilizadas na execução da preparação psicológica, como apontado pela litera86 tura (Costa Junior, 2005), o fornecimento de informação (via oral) apresenta-se como a técnica mais referida, seguido pela ludoterapia, técnica destinada especificamente ao público infantil. Entretanto, considerando que as intervenções de preparação psicológica baseadas unicamente no fornecimento de informação adequada e clara mostram-se menos eficazes do que quando envolvem uma combinação de outros procedimentos (Caminiti et al., 2005), uma parcela significativa dos psicólogos (40\%) afirmou utilizar, com frequência, outras técnicas na execução da preparação psicológica.

Por fim, no que se refere à avaliação dos resultados da intervenção, observa-se que apenas 36\% dos participantes (9) utilizam-se desse recurso como forma de verificar tanto o alcance dos objetivos traçados quanto a eficácia da técnica utilizada para alcançá-los, o que não contribui para o aumento do corpo de conhecimentos relacionados aos resultados positivos descritos na literatura, especialmente no tocante ao aumento dos índices de adesão ao tratamento (Caminiti et al., 2005), à redução de ansiedade antecipatória (Patel et al., 2006) e ao desenvolvimento de estratégias de enfrentamento mais eficientes (Franck \& Jones, 2003).

Entre os psicólogos que referiram realizar algum tipo de avaliação de resultados, observa-se que esta não se pautava em critérios claros, objetivos e/ou adequados para tal finalidade. Ressalta-se que, para serem eficazes e úteis, as avaliações das intervenções devem ser baseadas em evidências científicas, ou seja, provenientes de ações claras, objetivas e precisas, passíveis de serem compreendidas por outros profissionais de saúde (Gorayeb \& Guerrelhas, 2003).

\section{Considerações Finais}

Este estudo apresenta limitações no que se refere à não apreensão da realidade total dos serviços de psicologia pediátrica dos hospitais universitários públicos federais, tendo em vista o reduzido número de psicólogos que responderam aos convites para participar da pesquisa. Contudo, indica que, apesar de a preparação psicológica ser uma intervenção amplamente referida como utilizada, não há consenso, teórico ou prático, em relação a sua conceituação, nomenclatura, objetivos, técnicas e procedimentos de execução e avaliação. Faz-se necessário, portanto, o desenvolvimento de pes- 
quisas sobre a eficácia da preparação psicológica, aplicadas em diferentes contextos de cuidados com a saúde, e de momentos de tratamento, com diferentes fins, utilizando técnicas e/ou recursos variados que apontem relações funcionais mais precisas entre as intervenções e seus efeitos, a fim de estabelecer protocolos de ações práticas.

\section{Referências}

Blount, R. L., Piira, T., Cohen, L. L., \& Cheng, P.S. (2006). Pediatric procedural pain. Behavior Modification, 30 (1), 24-49.

Broering, C. V., \& Crepaldi, M. A. (2008). Preparação psicológica para a cirurgia em pediatria: importância, técnicas e limitações. Paidéia, 18 (39), 61-72.

Caminiti, C., Scoditti, U., Diodati, F., \& Passalacqua, R. (2005). How to promote, improve and test adherence to scientific evidence in clinical practice. BMC Health Services Research, 5 (62). Retrieved on January 1, 2006, available from: http://www.biomedcentral.com/1472-6963/5/62

Costa Junior, A. L. (2001). O desenvolvimento da psicooncologia: implicações para a pesquisa e intervenção profissional em saúde. Psicologia:Ciênciae Profissão, 21 (2), 36-43.

Costa Junior, A. L. (2005). Psicologia da saúde e desenvolvimento humano: o estudo do enfrentamento em crianças com câncer e expostas a procedimentos médicos invasivos. In M. A. Dessen \& A. L. Costa Junior (Orgs.), A essência do desenvolvimento humano: tendências atuais e perspectivas futuras (pp.171-189). Porto Alegre: Artmed.

Crepaldi, M. A., Rabuske, M. M., \& Gabarra, L. M. (2006). Modalidades de atuação do psicólogo em psicologia pediátrica. In M. A. Crepaldi, M. B. M. Linhares \& G. B. Perosa (Orgs.), Temas em psicologia pediátrica (pp.13-55). São Paulo: Casa do Psicólogo.

Doca, F. N. P., \& Costa Junior, A. L. (2007). Preparação psicológica para admissão hospitalar de crianças: uma breve revisão. Paidéia, 17 (37), 167-179.

Franck, L. S., \& Jones, M. (2003). Computer-taught coping techniques for venepuncture: preliminary findings from usability testing with children, parents and staff. Journal of Child Health Care, 7 (1), 41-54.

Gorayeb, R., \& Guerrelhas, F. (2003). Sistematização da prática psicológica em ambientes médicos. Revista Brasileira de Terapia Comportamental e Cognitiva, 5 (1), 11-19.
LeRoy, S., Elixson, E. M., O'Brien, P., Tong, E., Turpin, S., \& Uzark, K. (2003). Recommendations for preparing children and adolescents for invasive cardiac procedures. Circulation: Journal of The American Heart Association, 108 (20), 2550-2564.

Patel, A. D., Schieble, T., Davidson, M., Tran, M. C. J., Shoenberg, C., \& Delphin, E. (2006). Distraction with a hand-held video game reduces pediatric preoperative anxiety. Pediatric Anesthesia, 16 (10), 1019-1027.

Quiles, J. M. O., \& Carrillo, F. X. M. (2000). Hospitalización infanti: repercusiones psicológicas, teoria y práctica. Madrid: Editora Biblioteca Nueva.

Ribeiro, R. M., Tavano, L. A., \& Neme, C. M. B. (2002). Intervenções psicológicas nos períodos pré e pós-operatório com pacientes submetidos a cirurgia de enxerto ósseo. Estudos de Psicologia (Campinas), 19 (3), 67-76. doi: 10.159 0/S103-166×2002000300007.

Roberts, M. C. (2003). Handbook of pediatric psychology. New York: The Guilford Press.

Salmon, K. (2006). Commentary: Preparing young children for medical procedures: Taking account of memory. Journal of Pediatric Psychology, 31 (8), 859-861.

Soares, M. R. Z., \& Bomtempo, E. (2004). A criança hospitalizada: análise de um programa de atividades preparatórias para procedimento médico de inalação. Estudos de Psicologia (Campinas), 21 (1), 53-64. doi: 10.1590/S103$166 \times 200400100005$.

Tonetto, A. M., \& Gomes, W. B. (2007). A prática do psicólogo hospitalar em equipe multidisciplinar. Estudos de Psicologia (Campinas), 24 (1), 89-98. doi: 10.1590/S0103-166X2 007000100010.

Uman, L. S., Chambers, C. T., McGrath, P. J., \& Kisely, S. (2008). A systematic review of randomized controlled trials examining psychological interventions for needlerelated procedural pain and distress in children and adolescents: an abbreviated Cochrane Review. Journal of Pediatric Psychology, 33 (8), 842-854.

Yamada, M. O., \& Bevilacqua, M. C. (2005). O papel do psicólogo no programa de implante coclear do Hospital de Reabilitação de Anomalias Craniofaciais. Estudos de Psicologia (Campinas), 22 (3), 255-262. doi: 10.1590/\$10 3-166×200500300004.

Recebido em: 16/9/2009

Versão final reapresentada em: 12/4/2010

Aprovado em: 29/10/2010 